\title{
Photon Force-Induced Phase Transition Dynamics of Single Hydrogel Nanoparticles in Water
}

\author{
C. Hosokawa ${ }^{a, *}$, Y. Matsumura ${ }^{b, \dagger}$, H. Yoshikawa ${ }^{a}$, K. Iwai $^{b}$ \\ AND H. MASUHARA ${ }^{a, \ddagger, \S}$ \\ ${ }^{a}$ Department of Applied Physics, Osaka University \\ 2-1 Yamadaoka, Suita, Osaka 565-0871, Japan \\ ${ }^{b}$ Department of Chemistry, Nara Women's University \\ Kitauoya-Nishimachi, Nara 630-8506, Japan
}

Dedicated to Late Professor Jerzy Prochorow

\begin{abstract}
We investigated laser-induced phase transition dynamics of $\mu \mathrm{m}$ sized single $\operatorname{poly}(N$-isopropylacrylamide $)$ hydrogel particles in water. Single $\operatorname{poly}(N-$ -isopropylacrylamide) gel particles labeled with a polarity-sensitive fluorescent probe monomer were optically trapped by a focused laser beam and its fluorescence dynamics was analyzed. The fluorescence intensity of trapped single gel particles was increased with the trapping laser power, while the fluorescence peak wavelength was not changed. Temperature-induced changes of fluorescence properties of the single particle were confirmed to be similar to those of the bulk solution. These behaviors are well interpreted by considering that the fluorescence intensity and fluorescence peak reflect local interactions between the fluorescent probe and attaching (bound) water molecules and effective polarity determined by (free) water content in the particle, respectively. A change in the fluorescence peak wavelength after laser trapping was followed and its blue-shift was confirmed to occur within a few hundreds seconds, indicating that a single gel particle gradually attains to a globular state on this timescale, expelling initially free water molecules and then bound ones.
\end{abstract}

PACS numbers: 87.80.Cc, 33.50.Dq, 61.25.Hq, 87.64.--t

\footnotetext{
${ }^{*}$ Present address: Research Institute for Cell Engineering, National Institute of Advanced Industrial Science and Technology (AIST), 1-8-31 Midorigaoka, Ikeda, Osaka 563-8577, Japan.

${ }^{\dagger}$ Present address: Department of Materials and Life Science, Seikei University, 3-3-1 Kichijoji-Kitamachi, Musashino, Tokyo 180-8633, Japan.

${ }^{\ddagger}$ Present address: Hamano Life Science Research Foundation, TRI 309, 1-5-4 Minatojima-Minamimachi, Chuo-ku, Kobe 650-0047, Japan.

${ }^{\S}$ corresponding author; e-mail: masuhara@masuhara.jp
} 


\section{Introduction}

Laser has been contributing to the development of modern physical and chemical sciences from its invention in 1960. Scientists in molecular spectroscopy and photochemistry introduced lasers very early and used them as light sources for spectroscopic measurements as well as for inducing photophysical processes and photochemical reactions. Fundamental studies have started from isolated molecules in gas phase and in dilute solution, and then shifted to more complex molecules, molecular aggregates, polymers, and furthermore extended to colloids and molecular solids. In addition, the development of photochemistry from homogeneous to inhomogeneous systems has led to the combination of lasers with optical microscopes [1-4]. Time-resolved spectroscopy was extended to a time- and space-resolved one, while picosecond and femtosecond laser pulses have improved the temporal resolution. Such utilization of ultrashort lasers and microscopes brought about the experimental conditions of high intensity excitation, and consequently laser ablation has become gradually familiar to photophysicists and photochemists. The dynamics and mechanistic studies on ablation are scientifically important as they are expected to elucidate how electronic excitation of molecular materials evolves to their morphology changes. The other chemistry under high intensity laser beam is based on optical trapping potential exerted on $\mu \mathrm{m}$ sized particles and $\mathrm{nm}$ colloids under a microscope. Photochemistry using lasers in small space is indeed innovative and is being recognized laser nanochemistry [5]. Some of the main topics on which we have worked in the past quarter century are spectroscopy, photochemistry, ablation, and molecular trapping [6-8].

As a monochromatic light source, laser enables us to measure fine and precise spectra of molecules and to assign stable, excited, and transient molecular species; high resolution spectroscopy. Moreover, very specific reactions can be controlled by using a small difference in electronic energy levels, whose representative example is laser isotope separation. Another contribution of the laser is surely a high potential in developing time-resolved spectroscopy. It is well known that M. Eigen, R.G.W. Norrish, and G. Porter were awarded the Nobel Prizes for their work on fast reactions [9]. Their pump-probe experiments showed us how to interrogate the dynamic chemical reactions. In the flash photolysis by Norrish and Porter, molecular excited states were produced by the irradiation of an intense flash lamp, and then the succeeding radicals, ions, isomers, and/or unstable intermediates are spectroscopically detected by a probing flash lamp. This pump-probe approach is now a standard of the analysis of chemical reactions and has been introduced widely into various spectroscopies. The first laser oscillation was achieved using a ruby laser and its pulse duration was $10 \mathrm{~ns}$. Later on, the improvement of pulse width reduced up to picosecond in the late 1960s, to subpicosecond in the middle of the 1970s, until the femtosecond laser came in the early 1980s. The development was so fast and novel chemistry achieved by such short laser pulses has developed very quickly, which is symbolized by the Nobel Prize on femtochemistry awarded to A.H. Zewail [10]. 
Prochorow and one of the authors (H.M.) started our researches in the era when spectroscopists and photochemists started to use pulsed lasers. The first trial to introduce the pump-probe approach to optically scattering powders was done in 1981 by Kessler and Wilkinson [11], who proposed nanosecond diffuse reflection spectroscopy. Various kinds of powders as well as particles became research targets for transient absorption spectroscopy, so that their excited singlet and triplet states, neutral and ion radicals could be measured. Studies on organic microcrystals, photocatalytic semiconductor particles, zeolites, polymer particles, papers, textile fibers, and many scattering materials by time-resolved absorption spectroscopy were started, and their reported spectroscopic data were actually similar to those of homogeneous transparent systems. Prochorow and some members of Osaka University applied picosecond diffuse reflection spectroscopy to charge transfer crystal powder, revealed its primary processes for the first time [12], and thus contributed to the relevant research field.

Recently one of contemporary scientific interests is toward the laser-induced phenomena and we are demonstrating chemistry by laser trapping as its example. Here one representative experiment is presented, which is performed by applying optical trapping technique to elucidate phase transition phenomena. Stimuli-responsive polymer gels have attracted widespread interest in the past decades. These materials can change their volumes in response to their environmental condition (environmental stimuli), such as temperature, $\mathrm{pH}$, and concentration of additives. Poly $(N$-isopropylacrylamide) (PNIPAM) hydrogel is one of the most frequently studied temperature-responsive hydrogels [13]. Below the volume phase transition temperature $\left(\approx 34^{\circ} \mathrm{C}\right)$, PNIPAM hydrogels contain more water and are in a swollen state, whereas at higher temperature they expel water and are shifted to a globular state [14].

Scaling down the size of PNIPAM hydrogel is one of the contemporary topics and now microscopic particles known as microgels or nanogels are widely synthesized by conventional cross-linking of polymers. Recently, the microgel particles have attracted much attention in the fields of drug delivery systems, in situ thermosensors for biological tissue, fast optical switches, and so on $[15,16]$, since these particles have an advantage that response of volume change is faster with reduction of the gel size. The volume phase transition of PNIPAM gel particles in water has been evaluated using light scattering, turbidimetric analysis, differential scanning calorimetry, ${ }^{1} \mathrm{H}$ NMR, and so forth [17-20]. These studies reveal that the PNIPAM gel particles dispersed in water are in a swollen state at room temperature due to hydrogen bonding between solvent water and amide groups, i.e. $N$-isopropylacrylamide (NIPAM) units and crosslinker methylenebisacrylamide (MBAM) units [21]. We have observed that PNIPAM microgel particles dispersed in water gradually shrank with elevating temperature $\left(\approx 31^{\circ} \mathrm{C}\right)$ and understood using the fluorescent probe technique that the microenvironment inside the gel particles changed to hydrophobic [22]. In addition, the hydrophilicity inside the 
gel particles at swollen state could decrease with the increase in crosslinker concentration [23]. However, all these results are based on ensemble measurements which yield information only on average properties of gel particles and the phase transition behavior is rarely examined at single-particle level.

When a single laser beam is highly focused on polymers and nanoparticles dispersed in water, a number of polymer chains and nanoparticles can be assembled at the focal spot due to optical trapping. This optical trapping technique, so-called "optical tweezers" which is useful for manipulating neutral particles has been refined over the years $[24,25]$. The novel method utilizing optical trapping has achieved formation of various kinds of polymer assemblies, such as PNIPAM, poly $(N$-vinylcarbazole), and polyfluorene derivatives in solution [26-30]. We have chosen nanoparticle suspensions as a model for polymer solution, and revealed that larger clusters of nanoparticles were first formed in a focal spot and evolved to a large assembly [31, 32]. In PNIPAM assemblies, several studies by using fluorescence analysis and confocal Raman spectroscopy have presented that conformation of the polymer assemblies were strongly influenced by trapping laser power, supporting that the volume phase transition of PNIPAM assemblies can be induced by optical trapping [33]. Furthermore, not only in PNIPAM assemblies but also in the gels, $\mu \mathrm{m}$ sized polyacrylamide gels containing triphenyl-methane leuco derivatives show photoinduced rapid swelling responses [34]. Juodkazis et al. has demonstrated reversible shrinkage in $\mu \mathrm{m}$ sized PNIPAM gels due to optical trapping rather than local heating [35].

Now it is well accepted that optical trapping has a high potential in fabrication of noble polymer assemblies and reversible control of functional gels. For understanding molecular nature of optically trapped PNIPAM gels, not only conformation of the gels at a steady state, but also dynamic properties of the gels during irradiation of the trapping laser beam should be clarified from the standpoint of conformational change proceeding in the focal spot. In the present work, we investigate how single PNIPAM gel particles respond to optical trapping by fluorescence analysis. Single PNIPAM gel particles labeled with polarity-sensitive fluorescent probe monomers are analyzed and their temperature-responsive behavior and volume change dynamics are discussed.

\section{Experimental section}

The PNIPAM gel particles labeled with 4- $N$-(2-acryloyloxyethyl)- $N$ -methylamino-7- $N, N$-dimethylaminosulfonyl-2,1,3-benzoxadiazole (DBD-AE) as a polarity-sensitive fluorophore (chemical structure is shown in Fig. 1a) were synthesized as reported previously [23]. The ratio of NIPAM to DBD-AE units and NIPAM to MBAM units in the gel particles were $2500 / 1$ and 100/1, respectively. The average diameter of the synthesized gel particles was determined to be $58 \pm 1 \mathrm{~nm}$ by scanning electron microscopy (SEM; DB235, FEI Company). The diameter of the gel particles in water should be submicrometer because they absorb enough 


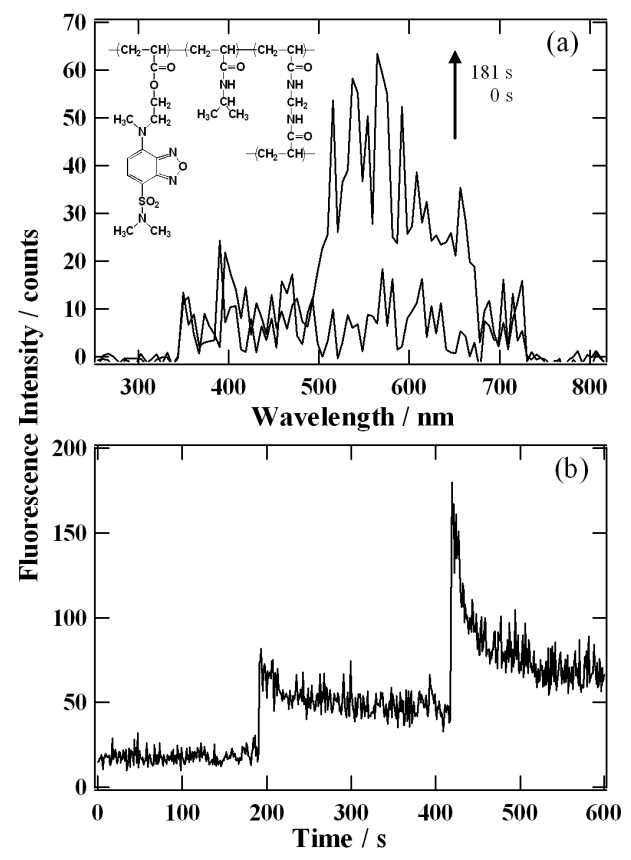

Fig. 1. Fluorescence spectra (a) and a temporal profile of the fluorescence intensity at the peak wavelength (b) of a single DBD-AE labeled PNIPAM gel particle in water. The inset in Fig. 1a shows the chemical structure of DBD-AE labeled PNIPAM gel. The particle concentration is $0.1 \mathrm{w} / \mathrm{v} \%$ in water. The trapping laser power is $90 \mathrm{~mW}$.

water inside the gel leading to the swollen state. The dried gel particles were dispersed in distilled water $(0.1 \mathrm{w} / \mathrm{v} \%)$ as a stock solution. The solution was diluted as required for the measurements.

A $1064 \mathrm{~nm}$ near-infrared (NIR) laser beam from a continuous wave (CW) $\mathrm{Nd}^{3+}$ :YAG laser (Spectron Laser Systems, SL902T1104) was used as a light source for optical trapping. A $405 \mathrm{~nm}$ visible laser beam from a diode pumped solid state laser (NEOARK, DPS-5001) was used as an excitation light source for fluorescence measurement. These laser beams were introduced to an inverted microscope (Carl ZEISS, Axiovert 200) and focused into a sample solution by an objective lens ( $\times 100$ magnification; numerical aperture 1.30 ). The spot diameters were $\approx 1 \mu \mathrm{m}$ for the optical trapping light source and $\approx 400 \mathrm{~nm}$ for the excitation light source. Both focal points were set at the same position. A thin layer of the solution was prepared by putting the dispersed solution between two glass plates with a $1 \mathrm{~mm}$ thickness silicone spacer. The fluorescence emission collected by the objective was passed through a notch filter (Kaiser Optical Systems) to remove scattered excitation light and then to a polychromator (Acton Research, Spectra Pro 300i) equipped with a charge-coupled device (CCD) camera (Roper Scientific, PIMAX1024HG18). The fluorescence spectra were measured with an exposure time 
of $1 \mathrm{~s}$. For the measurement of thermo-responsiveness, the sample temperature was monitored with a chromel-alumel thermocouple temperature probe.

\section{Results and discussion}

\subsection{Stepwise laser trapping}

When a $1064 \mathrm{~nm}$ laser beam for optical trapping and a $405 \mathrm{~nm}$ laser beam for photoexcitation were focused on DBD-AE labeled PNIPAM hydrogel dispersion, fluorescence spectra were observed as shown in Fig. 1a. Since the fluorescence spectrum around $600 \mathrm{~nm}$ was identified as that of DBD-AE unit, it appears that DBD-AE labeled PNIPAM gel particles were trapped at the focal point after a period of laser irradiation. When the trapping laser beam was turned off, the fluorescence spectrum became very weak and changed noisy, indicating that the trapped gel particles escaped from the focal spot. From fluorescence spectra which were acquired with $1 \mathrm{~s}$ exposure time, we obtained temporal profiles of the fluorescence intensity at peak wavelength $\lambda_{\max }$, as shown in Fig. 1b. The fluorescence intensity of PNIPAM gel particle dispersion increased stepwisely with the laser irradiation time. The mean fluorescence intensity of the firstly trapped gel was $51 \pm 3$ counts/s, which was obtained from several tens of measurements of the dispersion with particle concentration $(0.1$ to $0.01 \mathrm{w} / \mathrm{v} \%)$. Under the present condition, PNIPAM gel particles, which are spherical and exhibit narrow distribution of diameters as characterized by SEM images, are trapped one by one at a focal point. This is supported by the fact that the waiting time for observing the first step became longer as the concentration is lower. To investigate the properties and phase transition dynamics of the single gel particles, hereafter we acquired temporal profiles of fluorescence spectra at the lower concentration of $0.01 \mathrm{w} / \mathrm{v} \%$.

\subsection{Unconventional photobleaching}

Besides the stepwise increase, the fluorescence intensity was gradually decreased with the irradiation time of trapping laser, which is ascribed to photobleaching. Here it is noticeable that the photobleaching is not a conventional one-photon process but due to multiphoton process characteristic of laser trapping condition. Figure 2a shows the temporal profiles of the fluorescence intensity at the peak wavelength of a single gel particle from the beginning of optical trapping. It is interesting to see that the decreasing rate at high trapping laser power is faster than that at low one. While the fluorescence excitation of DBD-AE was held at $20 \mathrm{nW}$, the decreasing rate of the fluorescence intensity, i.e. the bleaching rate was determined by fitting the decay with a single exponential function. The rate increased with the increase in trapping laser power, as shown in Fig. 2b. Since the bleaching rate depends clearly on the trapping laser power, the photobleaching is enhanced by the simultaneous irradiations with trapping and fluorescence excitation lasers. A similar result has been reported by van Dijk et al., supporting that the enhanced photobleaching is caused by the ground state absorption of a visible photon followed by the resultant excited-state absorption of a near-infrared 


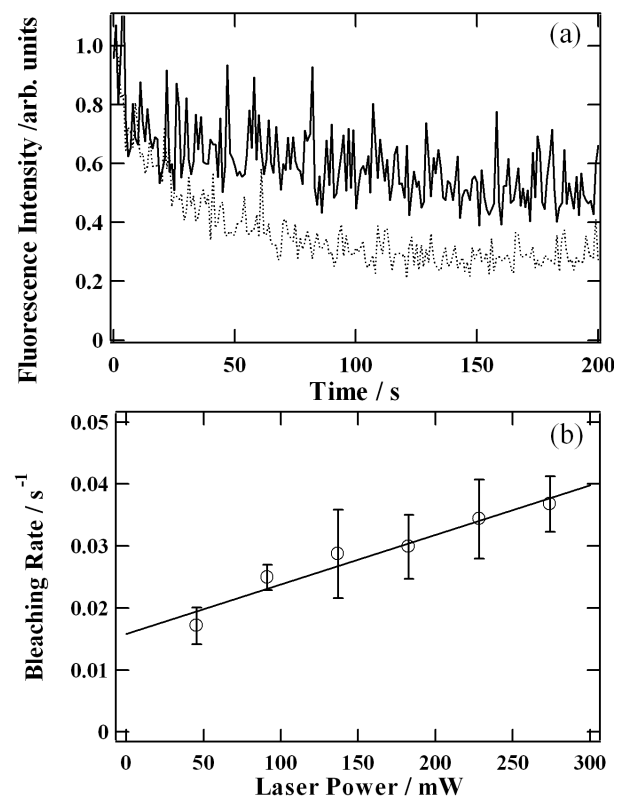

Fig. 2. Trapping laser power effect on photobleaching. (a) Temporal profiles of the fluorescence intensity of a single DBD-AE labeled PNIPAM gel particle from the beginning of optical trapping. The particle concentration is $0.01 \mathrm{w} / \mathrm{v} \%$ in water. The trapping laser power is $90 \mathrm{~mW}$ (solid line) and $270 \mathrm{~mW}$ (dotted line). (b) Dependence of the bleaching rate on trapping laser power. The average bleaching rate is obtained from $\approx 10$ particles at each power. Solid line shows a linear fit to the average data. The laser power of the $405 \mathrm{~nm}$ fluorescence excitation laser is $20 \mathrm{nW}$.

photon [36]. This explanation reminds us our mechanism that cyclic multiphoton absorption process of molecules during an excitation pulse is responsible to laser ablation. An excited state formed at the early part of the excitation pulse can absorb the following photon, and the formed higher excited state relaxes very rapidly $(\approx$ a few ps) to the first excited state through nonradiative deactivation. The latter state can again absorb the photon during the laser pulse, thus repeated absorption of excitation photons convert photon energy to thermal one very efficiently, resulting in photothermal ablation [37]. Similarly we consider that, in the present experiment, the higher excited state should undergo degradation, instead of nonradiative relaxation, which leads to the trapping power-dependent photobleaching. In the following analysis, the photobleaching is not regarded, since we notice to the fluorescence intensity of the first single gel particle just after its trapping.

\subsection{Phase transition by optical trapping}

It has been reported that its fluorescence peak wavelength $\left(\lambda_{\max }\right)$ of DBD-AE unit linked to the NIPAM, which is a polarity-sensitive fluorophore, was 

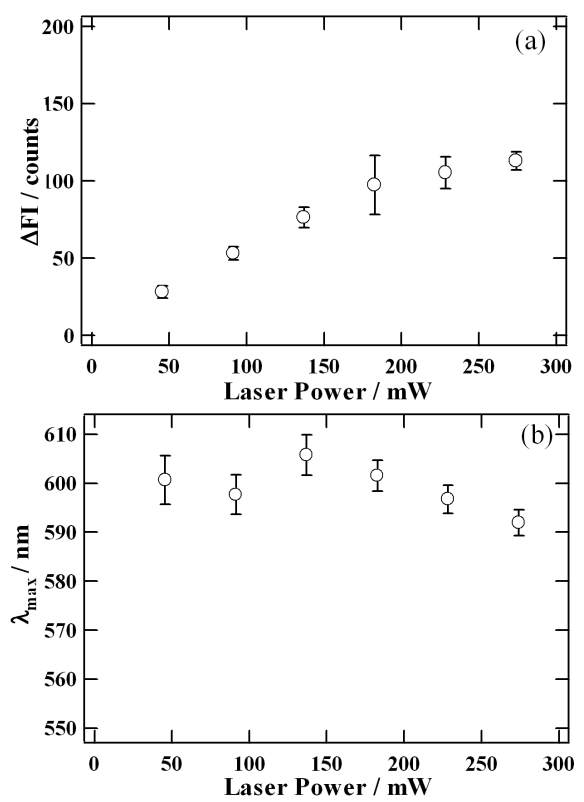

Fig. 3. The fluorescence intensity $\Delta \mathrm{FI}$ (a) and the fluorescence peak wavelength $\lambda_{\max }$ (b) of a single gel particle just after optical trapping as a function of trapping laser power. The $\Delta \mathrm{FI}$ and $\lambda_{\max }$ are acquired from the fluorescence spectrum just after the first particle is trapped in the gel particle suspensions. The particle concentration is $0.01 \mathrm{w} / \mathrm{v} \%$ in water.

blue-shifted and the fluorescence intensity was increased with the temperature-induced phase transition $[23,38]$. The increase in the fluorescence intensity $(\Delta \mathrm{FI})$, which is here the intensity difference between after and before the trapping, and blue-shift of the $\lambda_{\max }$ can be interpreted on the basis of the surrounding condition of DBD-AE units in the PNIPAM gel particle. The fluorophore is well known to show hydrophobic or hydrophilic environment which is closely related to contraction or swelling of the gel particle. Therefore, to evaluate the optical trapping effect on the conformational change of gel particles, we investigated $\Delta \mathrm{FI}$ and $\lambda_{\max }$ of trapped single gel particles. Figure 3 shows $\Delta$ FI and $\lambda_{\max }$ of the single gel particles just after the optical trapping $(<1 \mathrm{~s})$ as a function of trapping laser power. As shown in Fig. 3, $\Delta \mathrm{FI}$ correlated with the trapping laser power, while $\lambda_{\max }$ is virtually insensitive to the trapping laser power. In the conventional study on temperature-induced phase transition of bulk dispersion, a blue-shift of $\lambda_{\max }$ is observed with increasing $\Delta \mathrm{FI}$, so that the present result on single gel particles, apparently giving different behavior, will reveal more detailed properties and phase transition dynamics of hydrogels. For correlating the laser-induced fluorescence phenomenon of the single particle with the temperature-induced phase transition of the bulk dispersion, we examined temperature dependence on fluorescence prop- 
erties of single trapped gel particles. Immediately after the optical trapping of a single gel particle $(<1 \mathrm{~s})$ in aqueous solution on a temperature-controlled plate at $90 \mathrm{~mW}$ of the trapping laser power, $\Delta \mathrm{FI}$ and $\lambda_{\max }$ of the first step were examined and summarized in Fig. $4 . \Delta$ FI was constant and then reached $170 \pm 18$ counts $/ \mathrm{s}$ above $\approx 32^{\circ} \mathrm{C}$, while its $\lambda_{\max }$ gradually shifted from $600 \mathrm{~nm}$ to $560 \mathrm{~nm}$ and did not change anymore above $\approx 32^{\circ} \mathrm{C}$. The transition temperature of single gel particle is similar to that of bulk suspensions $\left(\approx 31.5^{\circ} \mathrm{C}\right)$, suggesting that the present trapping conditions (the laser power at $90 \mathrm{~mW}$ and the irradiation time within $1 \mathrm{~s}$ ) do not influence much the thermo-responsive behavior of the single gel particles. It is worth noting that the behaviors of $\Delta \mathrm{FI}$ and $\lambda_{\max }$ of single hydrogels are not in parallel with each other.

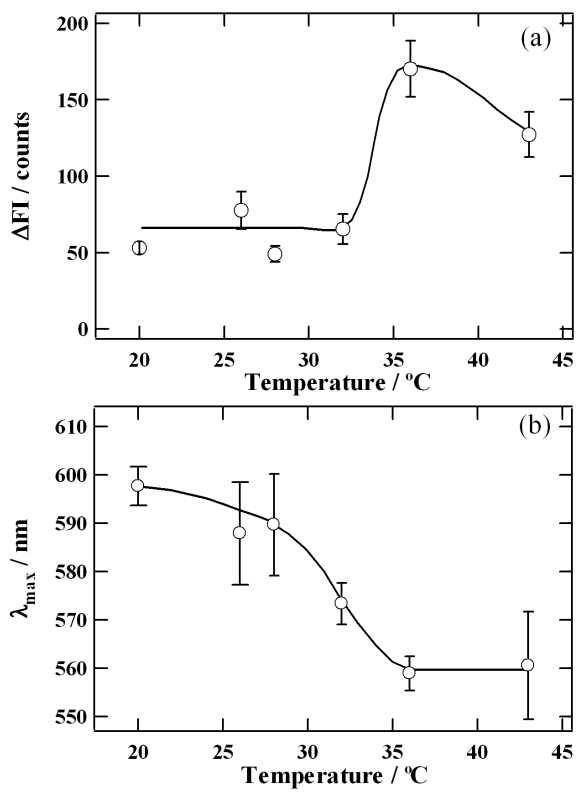

Fig. 4. Temperature dependence of the fluorescence intensity $\Delta \mathrm{FI}$ (a) and fluorescence peak wavelength $\lambda_{\max }$ (b) of a single gel particle just after optical trapping. The particle concentration is $0.01 \mathrm{w} / \mathrm{v} \%$ in water.

First we describe what parameters are responsible to $\Delta \mathrm{FI}$ and $\lambda_{\max }$. It is well known in general that molecules whose excited state have some electronic dipole moment show solvent polarity dependence, and in the present case hydrogel water content is crucial for effective polarity. Hydrogels containing enough water molecules are polar, but become hydrophobic above $\approx 32^{\circ} \mathrm{C}$, which has been interpreted in terms of its contraction in phase transition. Now we consider that not only water molecules attaching and oriented to the DBD-AE unit but also the surrounding water molecules around the unit determine the effective polarity, giving 
certain $\lambda_{\max }$. On the other hand, $\Delta \mathrm{FI}$ is dependent on radiative and nonradiative rate constants of the DBD-AE unit, and the former seems not to change largely as the shift in $\lambda_{\max }$ is not large compared to typical intramolecular charge transfer molecules. Thus it can be assumed that the nonradiative transition is important to understand the change in $\Delta \mathrm{FI}$ and the transition depends strongly on the local molecular interactions between attaching water molecules and the DBD-AE unit. Thus the present different behaviors between $\Delta \mathrm{FI}$ and $\lambda_{\max }$, summarized in Figs. 3 and 4, can be interpreted as follows. $\Delta \mathrm{FI}$ and $\lambda_{\max }$ are considered to reflect the short range intermolecular interaction between the DBD-AE unit and nearby water molecules, while $\lambda_{\max }$ is determined by the long range effective polarity.

According to the literatures on hydrogel dispersion, water molecules in the hydrogel have been deemed to be bound and free [39, 40]. The bound water was considered strongly associated with the polymer chains by hydrogen bonding or by dipole-dipole interactions, and free water has the properties similar to bulk water. The bound and free waters correspond to the attaching/oriented water molecules toward the DBD-AE unit and the surrounding ones described above, respectively. At room temperature hydrogel contains enough bound and free water molecules, while the latter is expelled and the hydrogel undergoes contraction from swollen state at higher temperature. Consequently its inside becomes hydrophobic and this temperature-induced phase transition is probed by polarity sensitive fluorophore. Now we consider how the traditional picture of the contraction and swelling states mutually switched by phase transition is extended to explain the single particle behavior. Some of the bound waters should be located to the DBD-AE unit, and may be responsible to the radiationless transition probability. Namely, $\Delta$ FI may be determined mainly by interactions with bound water molecules. On the other hand, $\lambda_{\max }$ is dependent on effective polarity, which is mainly ascribed to amount of free water in the hydrogel.

In Fig. 3b, $\lambda_{\max }$ is not clearly dependent on the laser power, so that the effective polarity is not changed, meaning that the content of water molecules in the hydrogel is almost common. As these data are obtained immediately after trapping, it is considered that free water cannot go out quickly from the gel. On the other hand, the increase in $\Delta \mathrm{FI}$ with the trapping laser power, shown in Fig. 3a, suggests the interactions between the bound waters and DBD-AE unit is affected by laser trapping. One possible explanation is that relative orientation between the unit and bound water molecules is changed by the intense linearly polarized laser irradiation. This reorientation does not need water flow in the hydrogel, so that even immediate measurement after the trapping gave clear laser power dependence. The results summarized in Fig. 4 can be interpreted in the same framework. As the laser power in this case is low $(90 \mathrm{~mW}), \Delta \mathrm{FI}$ is similar to that of the conventional bulk one. This is because the relative orientation between the DBD-AE unit and bound water molecules, which is responsible to the nonradiative transition, is common at the given swollen state below $\approx 32^{\circ} \mathrm{C}$. The shift in $\lambda_{\max }$ 
indicates that the free water content changes rather gradually in the temperature range around $32^{\circ} \mathrm{C}$, and then comes to contraction state above $\approx 32^{\circ} \mathrm{C}$.

\subsection{Phase transition dynamics}

Now we describe contraction dynamics of trapped single gel particles by analyzing the time trace of the $\lambda_{\max }$. Figure 5 shows the temporal profiles of $\lambda_{\max }$ in single PNIPAM gel particle from the beginning of optical trapping $(t=0 \mathrm{~s})$ to $250 \mathrm{~s}$. The $\lambda_{\max }$ was gradually blue-shifted with the irradiation time of a trapping laser after the single gel was trapped. The $\lambda_{\max }$ reached $580-565 \mathrm{~nm}$ at the irradiation time of $250 \mathrm{~s}$, which depended on the trapping laser power. The amount of blue-shift of $\lambda_{\max }$ per unit time, in other words, the rate of the blue-shift decreases with the increase in the trapping laser power. The higher trapping laser power more effectively induces the contraction of PNIPAM gel particle. It is noteworthy that the contraction is achieved by expelling free water molecules from the hydrogel. With regard to the contraction dynamics of a trapped gel particle at high laser power, the $\lambda_{\max }$ decreases to $\approx 565 \mathrm{~nm}$ at the irradiation time of $250 \mathrm{~s}$. It is worth noting that this wavelength is in good agreement with that of $560 \mathrm{~nm}$ above the transition temperature which is shown in the thermo-responsive measurement of trapped single gel particles (Fig. 4b). This means that the trapped single gel particle gradually changes to a globular state and undergoes phase transition within a few hundred seconds.

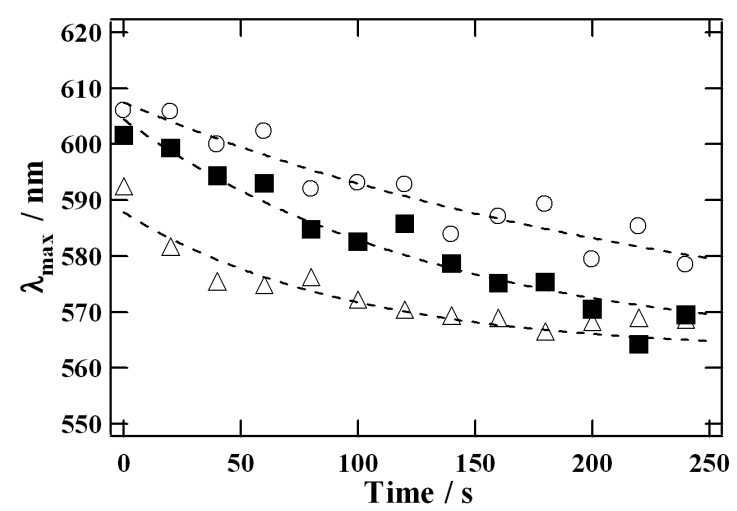

Fig. 5. Temporal profiles of the fluorescence peak wavelength $\lambda_{\max }$ of single gel particles from the beginning of optical trapping. The $\lambda_{\max }$ is averaged from about 5 data at each irradiation time. The trapping laser power is 90 (open circles), 180 (closed squares), and $270 \mathrm{~mW}$ (open triangles). Dashed line shows a single exponential fit to the average data. The rate constants of the blue-shift were obtained to be 250,140 , and $100 \mathrm{~s}$ with the irradiation of 90,180 , and $270 \mathrm{~mW}$ by fitting a single exponential, respectively.

Here, we discuss about optical trapping potential of a single PNIPAM gel particle. For optical trapping of a single gel particle, the depth of optical trapping potential energy $\left|U_{\text {trap }}\right|$ is required to be higher than the thermal energy $k T$, 
where $k$ is the Boltzmann constant and $T$ is the absolute temperature. $\left|U_{\text {trap }}\right|$ is represented as $\left|U_{\text {trap }}\right|=\alpha I_{0} n_{2} /\left(C \varepsilon_{2}\right)$, where $\alpha$ is the polarizability of the gel particle, $I_{0}$ is the laser intensity, $c$ is the speed of light, and $n_{2}$ and $\varepsilon_{2}$ are the refractive index and dielectric constant of the surrounding medium, respectively. $\alpha$ is given by $\alpha=4 \pi \varepsilon_{2} a^{3}\left[\left(n_{1} / n_{2}\right)^{2}-1\right] /\left[\left(n_{1} / n_{2}\right)^{2}+2\right]$, where $a$ is the radius of the particle and $n_{1}$ is the refractive index of the particle. In the case of a PNIPAM gel particle dispersed in water, since the diameter and the refractive index of the gel particle in the globule state are $58 \mathrm{~nm}$ as characterized by SEM images and 1.508 [27], $\left|U_{\text {trap }}\right|$ of the single gel particle is calculated to be $4.4 k T$ with a trapping laser power of $90 \mathrm{~mW}$, supporting that the single gel particle could be trapped at the focal point under the present condition.

Other possible factor for the laser-induced contraction of a single PNIPAM gel particle is heating effect due to the trapping laser beam. Recent studies have shown that the temperature rise was 10-20 $T$ (degree)/W on focusing a sub-W trapping laser in water [41-43]. In the case of our higher laser power $(270 \mathrm{~mW})$, the temperature rise is estimated to be $\approx 6 \mathrm{~K}$ at most, thus not reaching to the transition temperature. Actually the transition temperature of trapped single gel particles in water is in good agreement with that of the bulk dispersion as shown in Fig. 4. The heating effect is enough weak to have an influence on volume phase transition of the gel particle under the present conditions. In the effect of optical trapping potential which depends on laser power, it is noteworthy that the $\Delta \mathrm{FI}$ and the $\lambda_{\max }$ correlates with the trapping laser power and the irradiation time. This suggests that the cross-linked PNIPAM molecules in a single trapped gel particle are compacted at the focal point, because the optical trapping potential is approximated to Gaussian shape whose minimum is at the focal point. The molecular density in the gel particle should be highest at the center of the focal point and water exclusion is most prevailing. The dynamic aspect of these molecular features should be included in the contraction dynamics.

\section{Conclusion}

Optical trapping effect on single PNIPAM hydrogel particles labeled with a polarity-sensitive fluorescent probe in water was investigated by time-trace measurement of fluorescence spectra. We found that the single PNIPAM gel particle was trapped one by one at the focal spot. The conformational change of the gel particle was strongly correlated with the trapping laser power and the laser irradiation time. Immediately after optical trapping $(<1 \mathrm{~s})$, although $\Delta \mathrm{FI}$ increased with the trapping laser power, the $\lambda_{\max }$ was insensitive to it. Comparing the temperature-responsive behavior of trapped single gel particle with that of the bulk suspensions, we have explained the results in terms of bound and free waters in the hydrogel. Moreover, $\lambda_{\max }$ was blue-shifted with the laser irradiation time within a range of a few hundred seconds, during which the single gel particle in the trapping potential well gradually attains to the globular state. The present results clearly exhibit that the optical trapping can transiently change the PNIPAM 
gel structure in the focal spot. This trapping method will open the possibility of controlling internal states inside molecular assemblies and gels such as protein assembly in solutions, selective organelles in living cells.

\section{Acknowledgments}

One of authors (H.M.) wishes to express his sincere thanks to late Professor J. Prochorow for his long friendship from 1975 when they met in Tokyo and Osaka, and for his warm encouragement particularly when H.M. was starting his scientific career. This work was partly supported by a Grant-in-Aid for Scientific Research (KAKENHI) (S) (14103006) from the Japan Society for the Promotion of Science (JSPS).

\section{References}

[1] H. Masuhara, Pure Appl. Chem. 64, 1279 (1992).

[2] H. Masuhara, J. Photochem. Photobiol. A, Chem. 62, 397 (1992).

[3] H. Masuhara, N. Kitamura, H. Misawa, K. Sasaki, M. Koshioka, J. Photochem. Photobiol. A, Chem. 65, 235 (1992).

[4] Microchemistry: Spectroscopy and Chemistry in Small Domains, Eds. H. Masuhara, F.C. De Schryver, N. Kitamura, N. Tamai, North-Holland, Amsterdam 1994.

[5] H. Masuhara, T. Asahi, Y. Hosokawa, Pure Appl. Chem. 78, 2205 (2006).

[6] H. Masuhara, F.C. De Schryver, Organic Mesoscopic Chemistry (IUPAC 21st Century Chemistry Monograph), Blackwell Science, Oxford 1999.

[7] H. Masuhara, H. Nakanishi, K. Sasaki, Single Organic Nanoparticles, Springer, Berlin 2003.

[8] Nanophotonics: Integrating Photochemistry, Optics and Nano/Bio Materials Studies, Ed. H. Masuhara, S. Kawata, Elsevier, Amsterdam 2004.

[9] G. Porter, Chemistry in Microtime, Imperial College Press, London 1997.

[10] A.H. Zewail, Femtochemistry: Ultrafast Dynamics of the Chemical Bond, Vol. 1 and 2, World Scientific, Singapore 1994.

[11] R.W. Kessler, F. Wilkinson, J. Chem. Soc. Faraday Trans. I 77, 309 (1981).

[12] H. Fukazawa, H. Fukumura, H. Masuhara, J. Prochorow, Chem. Phys. Lett. 220, 461 (1994).

[13] H.G. Schild, Prog. Polym. Sci. 17, 163 (1992).

[14] S. Hirotsu, Y. Hirokawa, T. Tanaka, J. Chem. Phys. 87, 1392 (1987).

[15] L. Dong, A. Agarwal, D.J. Beebe, H. Jiang, Nature 442, 551 (2006).

[16] C.E. Reese, A.V. Mikhonin, M. Kamenjicki, A. Tikhonov, S.A. Asher, J. Am. Chem. Soc. 126, 1493 (2004).

[17] M.J. Murray, M.J. Snowden, Adv. Colloid. Interface Sci. 54, 73 (1995).

[18] C. Wu, S. Zhou, S.C.F. Au-Yeung, S. Jiang, Angew. Makromol. Chem. 240, 123 (1996).

[19] B.R. Saunders, B. Vincent, Adv. Colloid. Interface Sci. 80, 1 (1999). 
[20] R. Pelton, Adv. Colloid. Interface Sci. 85, 1 (2000).

[21] N.C. Woodward, B.Z. Chowdhry, M.J. Snowden, S.A. Leharne, P.C. Griffiths, A.L. Winnington, Langmuir 19, 3202 (2003).

[22] Y. Matsumura, K. Iwai, Polymer 46, 10027 (2005).

[23] Y. Matsumura, K. Iwai, J. Colloid Interface Sci. 296, 102 (2006).

[24] A. Ashkin, Proc. Natl. Acad. Sci. USA 94, 4853 (1997).

[25] A.G. Grier, Nature 424, 810 (2003).

[26] M. Ishikawa, H. Misawa, N. Kitamura, H. Masuhara, Chem. Lett. 22, 481 (1993).

[27] J. Hofkens, J. Hotta, K. Sasaki, H. Masuhara, K. Iwai, Langmuir 13, 414 (1997).

[28] P. Borowicz, J. Hotta, K. Sasaki, H. Masuhara, J. Phys. Chem. B 101, 5900 (1997).

[29] T.A. Smith, J. Hotta, K. Sasaki, H. Masuhara, Y. Itoh, J. Phys. Chem. B 103, 1660 (1999).

[30] S. Masuo, H. Yoshikawa, H.-G. Nothofer, A.C. Grimsdale, U. Scherf, K. Müllen, H. Masuhara, J. Phys. Chem. B 109, 6917 (2005).

[31] C. Hosokawa, Y. Yoshikawa, H. Masuhara, Phys. Rev. E 70, 061410 (2004).

[32] C. Hosokawa, Y. Yoshikawa, H. Masuhara, Phys. Rev. E 72, 021408 (2005).

[33] Y. Tsuboi, M. Nishino, T. Sasaki, N. Kitamura, J. Phys. Chem. B 109, 7033 (2005).

[34] M. Ishikawa, H. Misawa, N. Kitamura, H. Masuhara, M. Irie, Makromol. Chem. 12, 687 (1991).

[35] S. Juodkazis, N. Mukai, R. Wakaki, A. Yamaguchi, S. Matsuo, H. Misawa, Nature 408, 178 (2004).

[36] M.A. van Dijk, L.C. Kapitein, J. van Mameren, C.F. Schmidt, E.J.G. Peterman, J. Phys. Chem. B 108, 6479 (2004).

[37] H. Fukumura, H. Masuhara, Chem. Phys. Lett. 221, 373 (1994).

[38] S. Uchiyama, Y. Matsumura, A. Prasanna de Silva, K. Iwai, Anal. Chem. 75, 5926 (2003).

[39] F.X. Quinn, E. Kampff, G. Smyth, V.J. McBrierty, Macromolecules 21, 3191 (1988).

[40] X. Qu, A. Wirsen, A.-C. Albertsson, Polymer 41, 4589 (2004).

[41] S. Wurlitzer, C. Lautz, M. Liley, C. Duschl, T.M. Fischer, J. Phys. Chem. B 105, 182 (2001).

[42] E.J.G. Peterman, F. Gittes, C.F. Schmidt, Biophys. J. 84, 1308 (2004).

[43] S. Ito, T. Sugiyama, N. Toitani, G. Katayama, H. Miyasaka, J. Phys. Chem. B 111, 2365 (2007). 